\title{
Symmetry and Self-Duality in Categories of Probabilistic Models
}

\begin{abstract}
Alexander Wilce
This note adds to the recent spate of derivations of the probabilistic apparatus of finite-dimensional quantum theory from various axiomatic packages. We offer two different axiomatic packages that lead easily to the Jordan algebraic structure of finite-dimensional quantum theory. The derivation relies on the Koecher-Vinberg Theorem, which sets up an equivalence between order-unit spaces having homogeneous, self-dual cones, and formally real Jordan algebras.
\end{abstract}

\section{Introduction and Overview}

The last several years have seen a spate of derivations of the probabilistic apparatus of finite-dimensional quantum theory from various axiomatic packages, many having an information-theoretic motivation [7, 8, 10, 12, 13]. This note (which in part echoes, but greatly improves upon [19]) adds to the flow. I offer two different, though overlapping, axiomatic packages, both stressing symmetry principles, that lead quickly and easily to the Jordan algebraic structure of finite-dimensional quantum theory. Quickly and easily, at any rate, if one is familiar with the Koecher-Vinberg Theorem [11, 15], which sets up an equivalence between order-unit spaces having homogeneous, self-dual cones, and formally real Jordan algebras.

A probabilistic system can be described, in a standard way, in terms of an order-unit space $A$, the positive elements of which are scalar multiples of "effects". The strategy, then, is to show that certain strong, but not unreasonable, assumptions force the positive cone $A_{+}$to be homogeneous and self-dual, and hence, isomorphic to the cone of squares of such a Jordan algebra. In [3], several conditions are adduced that lead to a homogeneous and weakly self-dual cone - that is, a homogeneous cone that is order-isomorphic to its dual cone in $A^{*}$. However, proper self-duality is a much more stringent condition, requiring that the isomorphism be mediated by an inner product.

The line of attack here is to assume that systems individually have a great deal of symmetry, and collectively, can be organized into a symmetric monoidal category [1, 2, 14]. Here is a sketch. Further details can be found in the longer paper [20].

\section{Probabilistic Models}

A test space [16] is a pair $(X, \mathfrak{A})$ where $X$ is a set of outcomes and $\mathfrak{A}$ is a covering of $X$ by non-empty (for our purposes here, finite) subsets called tests, each understood as the set of possible outcomes of some measurement, experiment, etc. Two outcomes $x, y \in X$ are distinguishable iff they belong to a common test. In this case, I write $x \perp y$. Notice that there is, as yet, no linear structure in view, let alone an inner product; so this notation is promissory.

A state on a test space $(X, \mathfrak{A})$ is a function $\alpha: X \rightarrow[0,1]$, summing independently to unity on each test. A symmetry of $(X, \mathfrak{A})$ is a mapping $g: X \rightarrow X$ such that $g(E), g^{-1}(E) \in \mathfrak{A}$ for every $E \in \mathfrak{A}$. By a probabilistic model, I mean a structure $(X, \mathfrak{A}, \Omega, G)$ where $(X, \mathfrak{A})$ is a test space, $\Omega$ is a compact convex set of states on $(X, \mathfrak{A})$, and $G$ is a group acting on $(X, \mathfrak{A})$ by symmetries, and leaving $\Omega$ invariant.

Bart Jacobs, Peter Selinger, and Bas Spitters (Eds.):

8th International Workshop on Quantum Physics and Logic (QPL 2011)

EPTCS 95, 2012, pp. 275-279 doi 10.4204/EPTCS.95.19 
For illustration, if $\mathbf{H}$ is a finite-dimensional Hilbert space (real or Complex), the corresponding quantum model is $(X(\mathbf{H}), \mathfrak{A}(\mathbf{H}), \Omega(\mathbf{H}), U(\mathbf{H}))$, where $X=X(\mathbf{H})$ is the set of rank-one projection operators on $\mathbf{H}, \mathscr{A}=\mathfrak{A}(\mathbf{H})$ is set of (projective) frames, i.e., maximal pairwise orthogonal sets of projections, $\Omega(\mathbf{H})$ is the convex set of density operators on $\mathbf{H}$, and $U(\mathbf{H})$ is the group of unitary operators on $\mathbf{H}$, acting on $X(\mathbf{H})$ by conjugation.

Categories of Models. I will be interested in categories of models. A morphism from a model $(X, \mathfrak{A}, \Omega, G)$ to a model $(Y, \mathfrak{B}, \Gamma, H)$ is a pair $(\phi, \psi)$, where

(i) $\phi: X \rightarrow Y$ with $\phi(\mathfrak{A}) \subseteq \mathfrak{B}, \phi^{*}(\Gamma) \subseteq \Omega$

(ii) $\psi \in \operatorname{Hom}(G, H)$;

(iii) $\phi(g x)=\psi(g) \phi(x)$ for all $x \in X, g \in G$.

In what follows, $\mathscr{C}$ is a symmetric monoidal category of probabilistic models $A=(X(A), \mathfrak{A}(A), \Omega(A), G(A))$, with morphisms as above. I shall make two further assumptions:

(1) For every $A \in \mathscr{C}, G(A) \subseteq \mathscr{C}(A, A)$.

(2) The model $A \otimes B \in \mathscr{C}$ is a composite of the models $A, B \in \mathscr{C}$, in the sense of [5]. This means, in particular, that there are canonical injections $\otimes: X(A) \times X(B) \rightarrow X(A \otimes B)$ and $\otimes: \Omega(A) \times \Omega(B) \rightarrow$ $\Omega(A \otimes B)$, with

$$
E \otimes F=\{x \otimes y \mid x \in E, y \in F\} \in \mathfrak{A}(A \otimes B)
$$

for every $E \in \mathfrak{A}(A), F \in \mathfrak{A}(B)$, and

$$
(\alpha \otimes \beta)(x \otimes y)=\alpha(x) \beta(y)
$$

for all $\alpha \in \Omega(A), \beta \in \Omega(B), x \in X(A)$ and $y \in X(B)$. A bipartite state between $A, B \in \mathscr{C}$ is a state $\omega$ in $\Omega(A \otimes B)$. It is also part of the definition that the un-normalized conditional state $\hat{\omega}(x):=\omega(x, \cdot)$ belong to $\Omega(B)$ for every $x \in X$, and similarly with $A$ and $B$ reversed.

Models Linearized. Every model $A=(X(A), \mathfrak{A}(A), \Omega(A), G(A)) \in \mathscr{C}$ generates, in a standard and quite canonical way, an order-unit space $\mathbf{E}(A)$. To be precise, $\mathbf{E}(A)$ is the span in $\mathbb{R}^{\Omega}$ of the evaluation functionals associated with measurement outcomes $x \in X$.) In the case of a quantum model $A(\mathbf{H})=$ $(X(\mathbf{H}), \mathfrak{A}(\mathbf{H}), \Omega(\mathbf{H}), U(\mathbf{H}))$, one has $\mathbf{E}(A) \simeq \mathscr{L}(\mathbf{H})$, the space of Hermitian operators on $\mathbf{H}$, with the usual ordering and $u_{A}=\mathbf{1}_{\mathbf{H}}$.

The construction $A \mapsto \mathbf{E}(A)$ is functorial, so we obtain from $\mathscr{C}$ a category $\mathbf{E}(\mathscr{C})$ of order-unit spaces and positive linear mappings. It is natural to enlarge this to a category $\mathscr{E}$ in which each hom-set $\mathscr{E}(A, B)$ is an ordered linear space, and in which, e.g., $\mathscr{E}(I, A) \simeq \mathbf{E}(A)$. In what follows, I assume that such a "linearized" category $\mathscr{E}$ has been fixed.

\section{Bi-Symmetric Models}

To tighten this structure further, I now ask that every $A \in \mathscr{C}$ enjoy a property I call bi-symmetry.

Definition. A model $A \in \mathscr{C}$ is bi-symmetric iff

(i) $G(A)$ acts transitively on the pure states (that is, extreme points) of $\Omega(A)$,

(ii) $G(A)$ acts transitively on $\mathfrak{A}$, and on pairs $(x, y)$ of outcomes with $x \perp y$. 
If, in place of (ii), we require that arbitrary bijections $f: E \rightarrow F, E, F \in \mathfrak{A}$, extend to elements of $G$, then $A$ is fully bi-symmetric.

If $A$ is bi-symmetric, then $G$ acts transitively. Clearly, the quantum model discussed above is fully bi-symmetric. Bi-symmetry and full bi-symmetry, are very natural conditions. (See [17] for further discussion and motivation of the latter.)

Definition. A SPIN form ${ }^{1}$ for the model $A$ is a real bilinear form $B$ on $\mathbf{E}(A)$ that is symmetric, positive in the sense that $B(a, b) \geq 0$ for all $a, b \in \mathbf{E}(A)_{+}$, invariant, in the sense that $B(g a, g b)=B(a, b)$ for all $g \in G(A)$, and normalized, in the sense that $B\left(u_{A}, u_{A}\right)=1$. A SPIN form is orthogonalizing iff $B(x, y)=0$ for all distinguishable measurement outcomes $x, y \in X(A)$.

An example is the usual tracial inner product on $\mathscr{L}(\mathbf{H})$. Call $\mathbf{E}(A)$ irreducible iff (with respect to any SPIN form $B$ ), the subspace $u^{\perp}=\{a \in \mathbf{E}(A) \mid B(a, u)=0\}$ (this is independent of $B$ ) is irreducible under the group $G(A)$. Quantum models are irreducible in this sense.

Theorem 1. If $\mathbf{E}(A)$ is irreducible, it supports at most one orthogonalizing SPIN form, which - if it exists - is an inner product.

\section{Conjugates and Daggers}

At this point, the aim is to find sufficient conditions for the existence of an orthogonalizing SPIN form on $\mathbf{E}(A)$. I shall provide two.

Definition. By a conjugate for a model $A$, I mean a structure $\left(\bar{A}, \gamma_{A}, \eta_{A}\right)$, where $\gamma_{A}: A \mapsto \bar{A}$ is an isomorphism of models, and $\eta_{A}$ is a bipartite state on $A \times \bar{A}$ such that $\eta\left(x, \gamma_{A}(x)\right)=1 / n$ (where $n$ is the rank of $A$ ) for every $x \in X(A)$.

In the case of a quantum-mechanical model $A=A(\mathbf{H})$ associated with a Hilbert space $\mathbf{H}$, the obvious conjugate model is just that associated with the conjugate Hilbert space $\overline{\mathbf{H}}$, with $\gamma_{A}$ taking the rank-one projection $x$ to the corresponding projection $\bar{x}$ on $\overline{\mathbf{H}}$, and with $\eta_{A}$ the pure state associated with the unit vector $\frac{1}{\sqrt{n}} \sum_{i} e_{i} \otimes \bar{e}_{i},\left\{e_{i}\right\}$ any basis for $\mathbf{H}$ (note that this is basis-independent).

Returning to the general case, note that by averaging over $G(A)$, we can choose $\eta_{A}$ to be invariant, in the sense that $\eta_{A}\left(g x, \gamma_{A}(g y)\right)=\eta_{A}(g x, g y)$ for all $g \in G(A)$. This gives us an invariant SPIN form on $\mathbf{E}(A)$, defined on outcomes by $B(x, y):=\eta\left(x, \gamma_{A}(y)\right)$. Applying Theorem 1 , we have

Theorem 2. Let $\mathbf{E}(A)$ be irreducible, and suppose $A$ has a conjugate. Then $\mathbf{E}(A)$ carries a canonical orthogonalizing inner product.

Under some mild auxiliary hypotheses, the existence of a conjugate for every $A \in \mathscr{C}$ (with $\gamma_{A}$ and $\eta_{A}$ appropriately belonging to $\mathscr{C}$ ) can be used to construct a dagger on the category $\mathscr{E} \supseteq \mathbf{E}(\mathscr{C})$ discussed above. In fact, however, the mere existence of a reasonable dagger-monoidal structure on $\mathscr{E}$ is enough to obtain much the same result.

Theorem 3. Suppose $\mathscr{E}$ supports a dagger-monoidal structure, such that for every $g \in G(A), g^{\dagger}=g^{-1}$ (i.e., $g \in G(A)$ is "unitary"). If $\mathbf{E}(A)$ is irreducible, then it carries an orthogonalizing inner product.

In order to obtain the self-duality of $\mathbf{E}(A)_{+}$for an irreducible model $A$, it now suffices to assume either of two simple further conditions:

Theorem 4. Suppose that either

${ }^{1}$ This is probably not the best choice of terminology. 
(a) In the context of Theorem 2 A has a conjugate such that the state $\eta_{A}$ is an isomorphism state or

(b) In the context of Theorem $3 A$ is sharp, meaning that every outcome has probability one in a unique state on $\mathbf{E}(A)$.

Then $\mathbf{E}(A)_{+}$is self-dual.

The homogeneity of $\mathbf{E}(A)_{+}$can now be enforced by any of several conditions discussed in [4, 19]. Applying the Koecher-Vinberg Theorem, we can conclude that $\mathbf{E}(A)$ carries a unique Jordan product making it a formally real Jordan algebra.

One of these conditions is so simple it's worth pausing to describe it. Any bipartite state $\omega$ between $A, B \in \mathscr{C}$ gives rise to a natural positive linear mapping $\hat{\omega}: \mathbf{E}(A) \rightarrow \mathbf{E}(B)^{*}$, uniquely defined by $\hat{\omega}(x)(y)=$ $\omega(x, y)$. Where $\hat{\omega}$ is an order-isomorphism - that is, where $\hat{\omega}$ is an order-isomorphism (that is, invertible and having a positive inverse), we call $\omega$ an isomorphism state. A basic observation from [4], translated into the present context, is that if every state in the interior of $\Omega(B)$ is the marginal of an isomorphism state, then the cone in $\mathbf{E}(B)$ generated by $\Omega(B)$ is homogeneous.

\section{Image-Closure}

In order to extend these results to possibly reducible systems, I impose one further constraint on $\mathscr{C}$. Call a morphism $(\phi, \psi):(X, \mathfrak{A}, \Omega, G) \rightarrow(Y, \mathfrak{B}, \Gamma, H)$ is surjective iff $\phi(X)=Y, \mathfrak{B} \subseteq \phi(\mathfrak{A}), H=\psi(G)$, and $\Gamma=\left\{\beta \in \Omega(Y, \mathfrak{B}) \mid \phi^{*}(\beta) \in \Omega\right\}$. In this case, we call $(Y, \mathfrak{B}, \Gamma, H)$ the image of $(X, \mathfrak{A}, \Omega, G)$ under $(\phi, \psi)$. Call $\mathscr{C}$ image-closed iff, for any $A \in \mathscr{C}$ and any surjective morphism $(\phi, \psi):\left(X_{A}, \mathfrak{A}_{A}, \Omega_{A}, G_{A}\right) \rightarrow$ $(Y, \mathfrak{B}, \Gamma, H)$, (i) the model $B:=(Y, \mathfrak{B}, \Gamma, H)$ belongs to $\mathscr{C}$, and (ii) $(\phi, \psi) \in \mathscr{C}(A, B)$. in $\mathscr{C}$, again belong to $\mathscr{C}$.

Theorem 5. Let $\mathscr{C}$ be an image-closed category of bi-symmetric probabilistic models, and let $\mathscr{E}$ be the corresponding linearized category as discussed in Section 1. If either

(a) every $A \in \mathscr{C}$ has a conjugate $\bar{A} \in \mathscr{C}$, with $\eta_{A}$ an isomorphism state, or

(b) $\mathscr{E}$ has a dagger-monoidal structure making every $g \in G(A)$ unitary for all $A \in \mathscr{C}$, and every $A \in \mathscr{C}$ is sharp, then for every $A \in \mathscr{C}, \mathbf{E}(A)_{+}$is self-dual.

Again, adding any of the sufficient conditions for homogeneity from [4, 17] — or simply assuming it outright - will yield a category of formally real Jordan algebras.

Operationally, it is reasonable to suppose that any image $\phi(A)$ of a model $A \in \mathscr{C}$ can be simulated by means of the model $A$. Hence, if we wish to think of $\mathscr{C}$ as closed under operationally reasonable constructions, it is not far-fetched that $\phi(A)$ should belong to $\mathscr{C}$. In fact, the image of a bi-symmetric model is 2 -symmetric, so one can simply "close up" $\mathscr{C}$ without sacrificing this assumption. (To suppose that this closure continues to support, e.g., a symmetric-monoidal structure, or conjugate systems, is a sharper constraint, of course.) Categories of finite-dimensional quantum models turn out to be imageclosed for the simple reason that a quantum model has no non-trivial images.

\section{Conclusion}

These results raise any number of interesting questions. For one thing, it is possible that the assumptions are stronger than advertised, singling out a narrower class than formally real Jordan algebras. It is noteworthy that I have not had to assume that $\mathscr{C}$ 's monoidal product is locally tomographic. In fact, in a forthcoming paper with Howard Barnum [6], we show (using a result of Hanche-Olsen) that if $\mathscr{C}$ is 
a dagger-monoidal category of finite-dimensional order-unit spaces with homogeneous self-dual cones, then local tomography, plus the existence in $\mathscr{C}$ of a system having the structure of a qubit, implies that every $A \in \mathscr{C}$ is isomorphic to the Hermitian part of a finite-dimensional complex $C^{*}$ algebra.

\section{References}

[1] S. Abramsky and B. Coecke, A categorical semantics of quantum protocols. Proceedings of the 19th IEEE conference on Logic in Computer Science (LiCS'04). IEEE Computer Science Press (2004). doi $10.1109 /$ LICS.2004.1319636

[2] J. Baez, Quantum quandaries: a category-theoretic perspective. arXiv quant-ph/0404040 (2004).

[3] H. Barnum, R. Duncan and A. Wilce, Symmetry, compact closure, and dagger-compactness for categories of convex operational models. arXiv:1004.2920(2010), to appear in J. Phil. Logic.

[4] H. Barnum, P. Gaebler and A. Wilce, Ensemble Steering, weak self-duality and the structure of probabilistic theories. arXiv 0912.5532v2 (2009).

[5] H. Barnum and A. Wilce, Information processing in convex operational theories. ENTCS 270 (2011), 3-15. doi $10.1016 /$ j.entcs.2011.01.002.

[6] H. Barnum and A. Wilce, Local tomography, homogeneous self-dual cones, and the structure of quantum theory. To appear.

[7] G. Chiribella, G. M. D'Ariano, and P. Perinotti, Probabilistic theories with purification. Phys. Rev. A. 81 (2010), arXiv:0908.1583v5 (2009), and Informational derivation of quantum theory, arXiv:1011.6451 (2010).

[8] B. Dakic and C. Brukner, Quantum theory and beyond: is entanglement special? arXiv:0911.0695 (2009).

[9] J. Faraut and A. Koranyi, Analysis on Symmetric Cones. Oxford, 1994.

[10] L. Hardy, Quantum theory from five reasonable axioms. arXiv:quant-ph/0101012 (2001)

[11] M. Koecher, Die Geodätischen von Positivitätsbereichen. Math. Ann. 135 (1958), 384-432. doi:10.1007/BF01351796.

[12] L. Masanes, M. Müller, A derivation of quantum theory from physical requirements. New J. Phys., 13 (2011). doi:10.1088/1367-2630/13/6/063001 arXiv:1004.1483, 2011.

[13] J. Rau, On quantum vs. classical probability. Annals of Physics 324 (2009), 2622-2637. doi:10.1016/j.aop.2009.09.013, arXiv:0710.2119, 2007.

[14] P. Selinger, Dagger compact closed categories and completely positive maps. ENTCS 2007. doi:10.1016/j.entcs.2006.12.018.

[15] E. Vinberg, Homogeneous cones. Sov. Math. Dokl. 1 (1960), 787-790.

[16] A. Wilce, Test Spaces. In D. Gabbay, K. Engesser and D. Lehman (eds.), The Handbook of Quantum Logic, volume II, North-Holland, 2010.

[17] A. Wilce, Symmetry and composition in probabilistic theories. ENTCS 270 (2011), 191-207. doi:10.1016/j.entcs.2011.01.031 arXiv:0910.1527, 2009.

[18] A. Wilce, Formalism and interpretation in quantum theory. Foundations of Physics 40(4): 434-462, 2010. doi:10.1007/s10701-010-9410-x philsci:3794, 2008.

[19] A. Wilce, Four and a half axioms for finite-dimensional quantum theory. arXiv:0912.5530 (2009), to appear in a volume in memory of Itamar Pitowsky.

[20] A. Wilce, Symmetry, Self-Duality and the Jordan Structure of Quantum Mechanics. arXiv:1110.6607v1 (2011). 